\title{
Clinicobiological Profile and Management Outcomes of Male Subfertility
}

\author{
Kimassoum Rimtebaye ${ }^{*}$, Franklin Danki Sillong2, Arya Zarif Agah Tashkand1, \\ Mignagnal Kaboro ${ }^{1}$, Lamine Niang ${ }^{3}$, Serigne Magueye Gueye ${ }^{3}$ \\ ${ }^{1}$ National General Referral Hospital of N'Djamena, N'Djamena, Chad \\ ${ }^{2}$ Prostestant Hospital of N'Gaoundéré, N'Gaoundéré, Cameroon \\ ${ }^{3}$ General Hospital of Grand Yoff of Dakar, Dakar, Senegal \\ Email: *melinarim@yahoo.fr
}

Received 3 October 2015; accepted 13 November 2015; published 16 November 2015

Copyright (C) 2015 by authors and Scientific Research Publishing Inc.

This work is licensed under the Creative Commons Attribution International License (CC BY). http://creativecommons.org/licenses/by/4.0/

(c) () Open Access

\begin{abstract}
Introduction: We aim to determine the epidemiology, clinical, paraclinical and etiologic aspects and to evaluate the management of male subfertility in urology. Methodology: We conducted a retrospective descriptive study of 137 patients referred to the urology department for male subfertility over a period of 4 years. The analyzed variables were: clinical, paraclinical characteristics and the post therapeutic evolution. Results: The mean age was 38.41 years (range: 24 to 67 years). The semen analysis was normal in 18 patients. The semen analysis showed oligoasthenozoospermia $(45.3 \%)$ and total azoospermia $(24.8 \%)$. Disease history was represented by: gonorrhea $(27.7 \%)$, urogenital schistosomiasis $(13.2 \%)$ and hernia repair $(1.2 \%)$. The hormonal test showed abnormal hormone levels including: FSH $(43.8 \%)$, LH $(46.7 \%)$, testosterone $(36.5 \%)$, prolactin $(22.6 \%)$. The incriminated etiologic factors were: varicocele $(5.8 \%)$, smoking $(23.7 \%)$, alcohol intake $(25.4 \%)$ and sexually transmitted diseases $(42.3 \%)$. The treatment was medical in $67.9 \%$ and surgical in $32.1 \%$ of cases. After a year of follow up, $13.9 \%$ of patient's wives were pregnant. Conclusion: Male subfertility is common in Chad. The causes are numerous and diverse. The treatment is etiology based. A better management of sexually transmitted diseases and urogenital schistosomiasis is the best way to prevent it.
\end{abstract}

\section{Keywords}

Subfertility, Andrology, Hormone, Semen Analysis, Varicocele

\footnotetext{
"Corresponding author.
} 


\section{Introduction}

Over the world, an estimated $8 \%-15 \%$ of couples are subfertile [1]. In most cases (50\%), men are held responsible where only $40 \%$ are idiopathic [2]. In Africa, the rate is about $12 \%-21 \%$ [3]. The subfertility is the inability to conceive for at least one year after trying to have unprotected sex [4] [5].

Male subfertility, more than erectile dysfunction, is the leading cause of marital conflict and divorce among African couples where the main purpose of marriage is procreation. In Africa and especially in Chad, subfertile men rarely consult and readily blame their wives who have to prove that they are normal through multiple investigations in gynecology.

The man's responsibility in the subfertility of the couple was for centuries considered to be exclusively related to erectile dysfunction. There is often confusion between virility and fertility, impotence and subfertility. Sperm was discovered by Anton Van Leeuwenhoek in 1679 and the recognition of azoospermia as a cause of infertility was established in 1850 . To avoid incriminating women, male subfertility was considered by lawyers in 1966 [1]. The causes of azoospermia are diverse and often interconnected making the investigation and management challenging.

In order to contribute to the management and improvement of male subfertility in urology in N'Djamena, Chad, our study, first of its kind in our country, proposes to determine the clinical, biological, etiological characteristics of subfertility and to evaluate the management.

\section{Method and Patients}

This was a descriptive and prospective study of 137 male patients, aged 20 years and above, with a semen analysis showing a male subfertility. These patients were managed in the urology department for male subfertility from January 2010 to December 2013.The inclusion criteria were male patients aged 20 years or above referred for subfertility. These men had abnormal sperm and could not procreate. The reasons for this study were explained to the patients and they consented as their medical records files will be anonymously used for science. Data were collected and analyzed using SPSS 18.1; Mean and frequency calculations were performed. The variables considered were:

- Epidemiological: age, occupation, ethnicity.

- Clinical: medical history (smoking, alcohol, urogenital tuberculosis, urogenital schistosomiasis, diabetes, hypertension), previous surgery (hernia repair, hydrocele repair), general condition, secondary sexual characteristics, gynecomastia, varicocele classified according to Dublin Amelar.

- Paraclinical: urine culture, semen culture, semen analysis (sperm volume, viscosity, sperm count, number of motile sperm for the issue, type of abnormal semen analysis), hormonal balance (FSH, LH, prolactin, testosterone and other exams).

- Therapeutic: medical treatment (vitamin E, injectable androgen, oral androgen, antibiotic therapy), surgical treatment (varicocelerepair according to Ivanissevich, urethral reconstruction) and information on the periods of fecundity of their spouse.

- Outcome: patients were regularly seen in urology department every 3 months up to 18 months from the date of their discharge. The focus of the follow-up was to assess the semen analysis, the semen analysis, the patient's sexual activity and the existence of pregnancy for the spouses.

These variables were obtained using a predefined questionnaire on the basis of files for consultation, hospitalization, operative report and outpatient follow-up.

\section{Results}

Mean age of patients was 38.41 years (Range: 24 to 67 years). The age groups 36 - 45 years and 26 - 35 years were the most represented, respectively in $41.6 \%$ and $34.3 \%$ of cases (Figure 1). Arabs were the most affected (21.9\%). Civil workers represented $63.5 \%$ followed by traders $21.2 \%$. Married men represented $76.6 \%$ of cases. Primary subfertility was found in $59.9 \%$ of cases. Subfertility was associated with erectile dysfunction in $64.2 \%$. A history of gonococcal urethritis (Figure 2) was implicated in $27.7 \%$ of cases, chlamydia trachomatis in $10.2 \%$, varicocele in (5.8\%) (Figure 3), smoking (23.7\%), alcohol intake (25.4\%) and sexually transmitted diseases (42.3\%). urogenital schistosomiasis in (13.2\%) (Figure 4) and hernia repair in (12.2\%). No comorbidity was identified in $81.8 \%$ of patients. Patients practiced 3 - 4 sexual intercourses a week. The general status was normal 


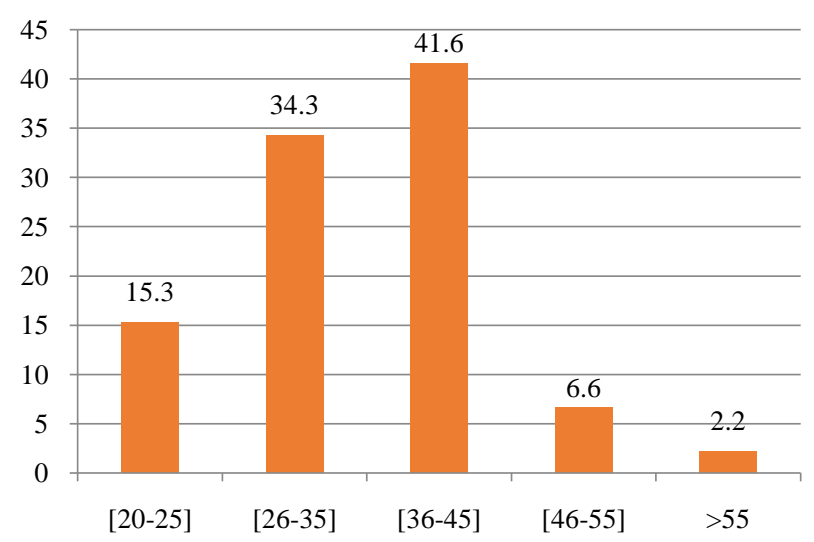

Figure 1. Distribution of patients by age.

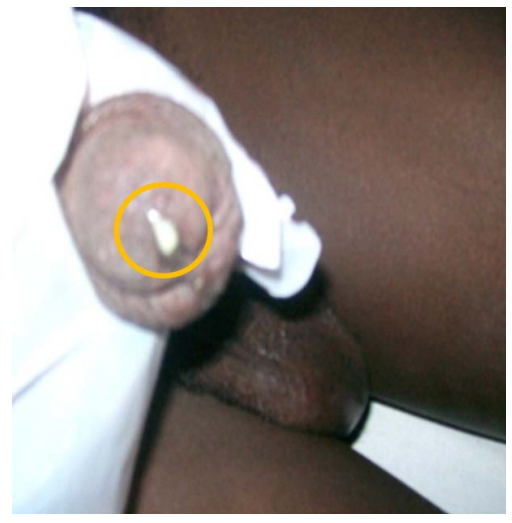

Figure 2. Suppurative gonococcal urethritis.

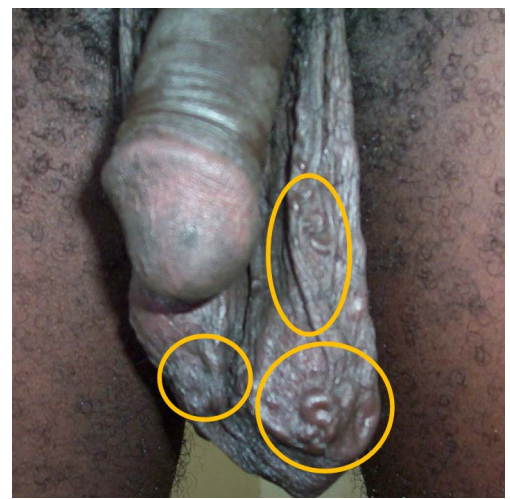

Figure 3. Bilateral varicocele clinically apparent from afar.

in $97.1 \%$ of patients and there was no gynecomastia in $95.6 \%$ of patients. Prostatitis was reported in $23.4 \%$ of cases. Semen analysis and semen culture results are reported in Table 1 and Figure 5. Patients had a normal LH (53.3\%), normal plasma testosterone (63.5\%) normal prolactin level (46.7\%). Chlamydia trachomatis serology was positive in $32.1 \%$ of patients. Urine culture showed a urinary tract infection in $59.9 \%$ of patients. Spouses of $13.9 \%$ of patients became pregnant during the follow-up period. Improvement in semen analysis was achieved in 37\% of cases. The open surgery according to Ivanissevich has been used in patients with varicocele, only $22.7 \%$ have procreated. Praziquantel has been used in patients with bilharzias calcifications but without success. Sex intercourse was improved for $35.8 \%$ of patients who have been associated with erectile dysfunction. 


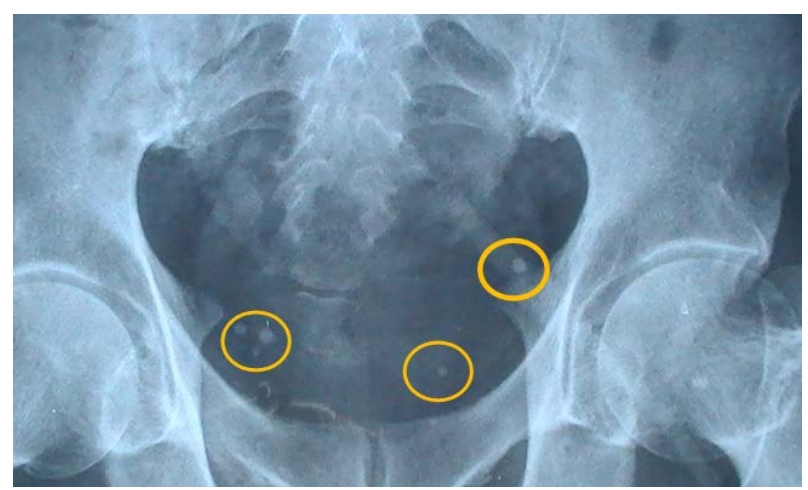

Figure 4. Bilharzia calcifications of the bladder area.

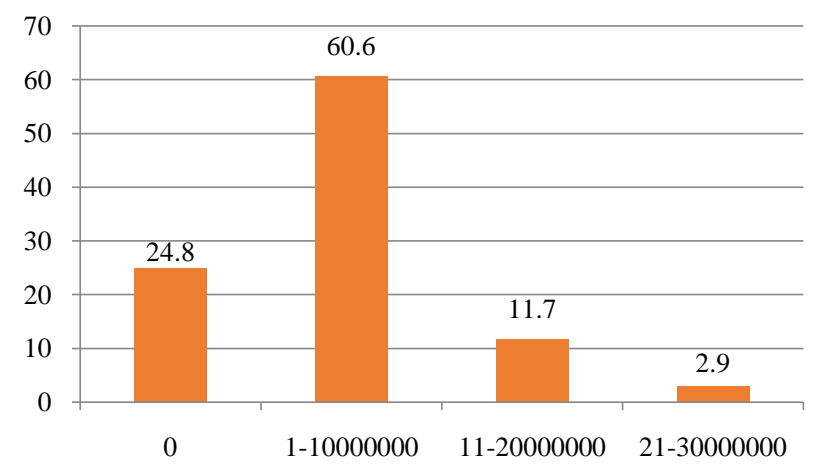

Figure 5. Distribution by semen analysis results.

Table 1. Distribution by the conclusion of the spermiogram reading.

\begin{tabular}{ccc}
\hline Spermiogram abnormalities & Number & Percent (\%) \\
\hline Total azoospermia & 34 & 24.8 \\
Necrozoospermia & 11 & 8 \\
Oligoasthenozoospermia & $\mathbf{6 2}$ & $\mathbf{4 5 . 3}$ \\
Oligozoospermia & 12 & 8.8 \\
Normal spermocytogram & 18 & 13.1 \\
Total & 137 & 100.0 \\
\hline
\end{tabular}

\section{Discussion}

In four years, 137 patients had consulted for male subfertility, 40.1\% were in the age group of 36 - 45 which corresponds to asexually active age range and resumed fertile population. This group is exposed to many conditions that can impair fertility: urogenital schistosomiasis, gonococcal urethritis, infection by Chlamydia tracomatis and herniorrhaphy. These conditions affect male fertility through various mechanisms. Abnormal spermatogenesis by destruction of the germ cell line, excretory azoospermia by post infectious scar stenosis of sperm ducts, iatrogenic lesions of the vas at the inguinal hernia repair. We must also add intoxication by alcohol, smoking and varicocele, where Varicocele reduces sperm mobility limiting their ability to reach the ovocyte. Jallouli M et al. report that cryptorchidism is the most common cause of male subfertility [6].

The role of urogenital schistosomiasis in subfertility is not reported by researchers from developed countries because this parasitic disease is not common in Europe while it is endemic in Central Africa and Chad in particular. The parasite enter the skin in contact with stagnant freshwater infested by cercariae. The aberrant location 
(vas deferens, epididymis, seminal vesicle...) of living eggs causes ulcers of various length, then the healing process causes obstruction of the genital tract, leading to an excretory azoospermia [7] [8]. The high concentration of Civil workers in the capital city of N'Djamena that host the only department of Urology in Chad explains the highest number of affected men among Civil workers compared to breeders and farmers in our study. The prolonged sitting of civil workers can cause testicular warming and harmful blood circulatory deficit on spermatogenesis. For Alon Y. Mass et al. [9] who comparedtaxi drivers (seated all day long to drive) and ordinary population concludes that there is a high percentage of infertility among taxi drivers compared with nonprofessional drivers; this is called taxi cab syndrome. Patients with primary subfertility (59.9\%) consult earlier than those with secondary subfertility who accuse their wives arguing that they have already had children. Among our subfertile patients, $35.8 \%$ also suffered from erectile dysfunction described as weakness of rigidity of erections. The inability to perform normal regular sex explains subfertility in this group of patients. For Shindel et al. [7], 22\% of subfertile men present moderate to severe erectile dysfunction. According to Berger, among men with azoospermia, more than half showed erectile dysfunction but never had trouble before [10]. High levels of FSH found in $27 \%$ of patients were in favor of excretory azoospermia observed in our patients. Testosterone rate was lower in patients with gynecomastia where there has been noticed hyperprolactinemia associated with oligoasthenozoospermia. Only $13.9 \%$ of managed subfertile men achieved a pregnancy in the year of treatment. This low success rate should prompt to better manage patients and to continue the investigation, treatment and monitoring. Prevention requires better management of STIs and urogenital schistosomiasis. The assessment must be completed in another study including chemical assay (zinc, carnitine, fructose ...) which implies improving the technical infrastructure of our laboratory.

The treatment will be most effective when it takes into consideration the contribution of zinc, carnitine, and fructose. It is recalled that the sperm will do a long trip which will go from testis, vas deferens, urethra, vagina, uterus, fallopian tubes, before they fertilize the egg. The sperm liquid supplied by the seminal vesicles and prostate gland must be rich in zinc, fructose and carnitine to supplement the sperm maturation process and bring the energy to achieve their goal: fertilize the egg. Some preventable causes such as alcohol and tobacco must be an information and awareness campaign through the communication for behavior change.

\section{Conclusions}

In four years, 137 men were consulted in the department of urology for subfertility. These patients underwent a treatment and a follow-up of at least two years.

The subfertlility was associated with erectile dysfunction in $64.2 \%$. Civil workers represented $63.5 \%$ of patients. The etiologies found were: gonorrhea (27.7\%), trachomatis chlamydia (10.2\%), urogenital schistosomiasis, inguinal hernia repair (12.2\%) and varicocele (5.8\%). Smoking was reported in 23.7\% of patients and alcohol intake in $25.4 \%$. Semen analysis showed oligoasthenozoospermia (47.4\%), total azoospermia (20\%) and decreased sperm count of less than 2000/ml (65\%). Only $13.9 \%$ of patients reported a pregnancy.

Male subfertility is common in Chad. To prevention it, we must go through prompt management of STIs and urogenital schistosomiasis. Improvement of the management requires etiological research that involves the improvement of the technical platform of the biochemistry laboratory.

\section{References}

[1] Agarwal, A., Mulgund, A., Hamada, A. and Chyatte, M.R. (2015) A Unique View on Male Infertility around the Globe. Reproductive Biology and Endocrinology, 13, 9 p.

[2] Li, H-J. (2014) More Attention Should Be Paid to the Treatment of Male Infertility with Drugs-Testosterone: To Use It or Not? Asian Journal of Andrology, 16, 270-273. http://dx.doi.org/10.4103/1008-682X.122343

[3] Methorst, C. and Huyghe, E., Committee members of Andrology and Sexual Medicine of the French Association of Urology, Subcommittee Male fertility CAMS (2014) Oxidative Stress and Male Infertility: Pathophysiology and Therapeutic Interest of Antioxidants (Stress oxydant et infertilité masculine: Physiopathologie et intérêtthérapeutique des antioxydants). Progrès en Urologie, 24, 4-10.

[4] Tarín, J.J., García-Pérez, M.A., Hamatani, T. and Cano, A. (2015) Infertility Etiologies Are Genetically and Clinically Linked with Other Diseases in Single Meta-Diseases. Reproductive Biology and Endocrinology, 13, 11 p. http://dx.doi.org/10.1186/s12958-015-0029-9

[5] Ammar, T., Sidhub, P.S. and Wilkins, C.J. (2012) Male Infertility: The Role of Imaging in Diagnosis and Management. 
The British Journal of Radiology, 85, S59-S68. http://dx.doi.org/10.1259/bjr/31818161

[6] Jallouli, M., Mefteh, S., Rebai, T. and Mhiri, R. (2010) Cryptorchidism: Effect on Fertility of Age at the Time of Orchidopexy, the Seat and the Size of the Testis and Epididymis of the Existence of Anomalies (Cryptorchidie: Influence sur la fertilité de l'âge au moment de l'orchidopexie, du siège et de la taille du testicule et de l'existenced' anomalies épididymaires). Andrologie, 20, 243-246. http://dx.doi.org/10.1007/s12610-010-0107-y

[7] Rimtebaye, K., Niang, L., Ndoye, M., Traore, I., Vadandi, V., Gueye, S.M. et al. (2014) Fournier’Sgangrene: Epidemiological, Clinical, Diagnostic and Therapeutic Aspects in Urology Department of N'Djamena (Gangrène de Fournier: Aspect épidémiologique, clinique, diagnostique et thérapeutique au service d’Urologie de N'Djamena). Revue Africained'Urologie et d'Andrologie, 1, 91-98.

[8] Shindel, A.W. Nelson, C.J., Naughton, C.K. and Muhall, J.P. (2008) Premature Ejaculation in Infertile Couples: Prevalence and Correlates. The Journal of Sexual Medicine, 8, 485-491. http://dx.doi.org/10.1111/j.1743-6109.2007.00690.x

[9] Mass, A.Y., Goldfarb, D.S. and Shah, O. (2014) Taxi Cab Syndrome: A Review of the Extensive Genitourinary Pathology Experienced by Taxi Cab Drivers and What We Can Do to Help. Reviews in Urology, 16, 99-104.

[10] Shindel, A.W. Nelson, C.J., Naughton, C.K., Ohebshalom, M. and Muhall, J.P. (2008) Sexual Function and Quality of Life in the Male Partner of Infertile Couples: Prevalence and Correlates of Dysfunction. Journal of Urology, 179, 10561059. http://dx.doi.org/10.1016/j.juro.2007.10.069 